\title{
An Advanced Version of the Local-Global Step Size Control for Runge-Kutta Methods Applied to Index 1 Differential-Algebraic Systems
}

\author{
Gennady Y. Kulikov \\ School of Computational and Applied Mathematics, University of the Witwatersrand, \\ Private Bag 3, Wits 2050, Johannesburg, South Africa \\ gkulikov@cam.wits.ac.za
}

\begin{abstract}
In recent paper 5] a procedure was developed to control a step size for Runge-Kutta methods. Here, we present a new version of that step size selection to make it better in exceptional cases when the old version does not work appropriately.
\end{abstract}

In this paper, we consider a system of differential-algebraic equations (DAEs) of the form

$$
x^{\prime}(t)=g(x(t), y(t)), \quad y(t)=f(x(t), y(t)),
$$

where $t \in[0, T], x(t) \in \mathbf{R}^{m}, y(t) \in \mathbf{R}^{n}, g: D \subset \mathbf{R}^{m+n} \rightarrow \mathbf{R}^{m}, f: D \subset \mathbf{R}^{m+n} \rightarrow$ $\mathbf{R}^{n}$, and where an initial conditions $x(0)=x^{0}, y(0)=y^{0}$ are also given and they are consistent; i. e., $y^{0}=f\left(x^{0}, y^{0}\right)$. We suppose that problem (1) possesses an unique solution $z(t) \stackrel{\text { def }}{=}\left(x(t)^{T}, y(t)^{T}\right)^{T}$ on the interval $[0, T]$.

To solve problem (1) numerically, we applied an $l$-stage Runge-Kutta (RK) method and come to the following discrete system:

$$
\begin{gathered}
x_{k i}=x_{k}+\tau_{k} \sum_{j=1}^{l} a_{i j} g\left(x_{k j}, y_{k j}\right), \quad y_{k i}=f\left(x_{k i}, y_{k i}\right), \quad i=1,2, \ldots, l, \\
x_{k+1}=x_{k}+\tau_{k} \sum_{i=1}^{l} b_{i} g\left(x_{k i}, y_{k i}\right), y_{k+1}=f\left(x_{k+1}, y_{k+1}\right), k=0,1, \ldots, K-1,
\end{gathered}
$$

We assume further that the RK formula is of order $s$, and that problem (1) satisfies the smoothness condition and the nonsingularity one in a neighborhood of the solution $z(t)$ on the interval $[0, T]$ (we refer to [5] for details).

It is well-known [1], 2], [3] that under the conditions mentioned above problem (1) is of index 1 and method (2) is convergent of order s. Moreover, it was proved in 3. that the local error of method (2) has the form

$$
\begin{aligned}
& x\left(t_{k+1}\right)-\tilde{x}_{k+1}=\check{\psi}_{s+1}\left(t_{k}\right) \tau_{k}^{s+1}+O\left(\tau_{k}^{s+2}\right), \\
& y\left(t_{k+1}\right)-\tilde{y}_{k+1}=\check{\phi}_{s+1}\left(t_{k}\right) \tau_{k}^{s+1}+O\left(\tau_{k}^{s+2}\right),
\end{aligned}
$$


$k=0,1, \ldots, K-1$, as $\tau_{k} \rightarrow 0$, where $\tilde{z}_{k+1} \stackrel{\text { def }}{=}\left(\left(\tilde{x}_{k+1}\right)^{T},\left(\tilde{y}_{k+1}\right)^{T}\right)^{T}$ is the solution of (2) provided that $z_{k}=z\left(t_{k}\right)$, and the functions $\check{\psi}_{s+1}\left(t_{k}\right), \check{\phi}_{s+1}\left(t_{k}\right)$ denote the coefficients of leading terms of the local error of RK method (2). In addition, Theorem 2.1 in 4 establishes that the global error of method (2) possesses the following asymptotic expansion:

$$
\begin{aligned}
& x\left(t_{k+1}\right)-x_{k+1}=\psi_{s}\left(t_{k+1}\right)\left(\tau_{k}^{*}\right)^{s}+O\left(\left(\tau_{k}^{*}\right)^{s+1}\right), \\
& y\left(t_{k+1}\right)-y_{k+1}=\phi_{s}\left(t_{k+1}\right)\left(\tau_{k}^{*}\right)^{s}+O\left(\left(\tau_{k}^{*}\right)^{s+1}\right),
\end{aligned}
$$

$k=0,1, \ldots, K-1$, as $\tau_{k}^{*} \rightarrow 0$, where the pair of functions $\psi_{s}(t)$ and $\phi_{s}(t)$ is a solution of the DAEs

$$
\begin{gathered}
\psi_{s}^{\prime}(t)=\partial_{x} g(x(t), y(t)) \psi_{s}(t)+\partial_{y} g(x(t), y(t)) \phi_{s}(t)+\check{\psi}_{s+1}(t), \\
\phi_{s}(t)=\partial_{x} f(x(t), y(t)) \psi_{s}(t)+\partial_{y} f(x(t), y(t)) \phi_{s}(t) \\
\psi_{s}(0)=0, \quad \phi_{s}(0)=0 .
\end{gathered}
$$

The new step size $\tau_{k}^{*}$ in formulas (4) means a step size for integrating problem (1) in the local interval $\left[t_{k}, t_{k+1}\right]$ by a fixed step size version of method (2). Below, we will discuss how to define it more precisely.

Taking into account possible stiffness of problem (5), we apply the implicit Euler method to this problem and obtain the following recursion relation for an approximate computation of the functions $\psi_{s}(t)$ and $\phi_{s}(t)$ :

$$
\begin{gathered}
\left(\begin{array}{c}
\tilde{\psi}_{s}\left(t_{k+1}\right) \\
\tilde{\phi}_{s}\left(t_{k+1}\right)
\end{array}\right)=P_{k+1}^{-1}\left(\begin{array}{c}
\tilde{\psi}_{s}\left(t_{k}\right)+\tau_{k} \hat{\psi}_{s+1}\left(t_{k+1}\right) \\
0
\end{array}\right), \\
\tilde{\psi}_{s}(0)=0, \quad \tilde{\phi}_{s}(0)=0, \quad k=0,1, \ldots, K-1,
\end{gathered}
$$

where

$$
P_{k+1} \stackrel{\text { def }}{=}\left(\begin{array}{cc}
I_{m}-\tau_{k} \partial_{x} g\left(\tilde{x}_{k+1}, \tilde{y}_{k+1}\right) & -\tau_{k} \partial_{y} g\left(\tilde{x}_{k+1}, \tilde{y}_{k+1}\right) \\
-\partial_{x} f\left(\tilde{x}_{k+1}, \tilde{y}_{k+1}\right) & I_{n}-\partial_{y} f\left(\tilde{x}_{k+1}, \tilde{y}_{k+1}\right)
\end{array}\right) .
$$

In formula (6a), 0 denotes the null vector of dimension $n$, but full particularities can be found in $[5$.

The next important stage in the numerical integration of semi-explicit DAEs of index 1 is a selection of the maximum step size keeping the truncated local and global errors bounded. Let $\epsilon_{l}$ and $\epsilon_{g}$ be given limits for the local and global errors of method (2), respectively. Considering that the global error of the numerical solution at the point $t_{k}$ does not exceed $\epsilon_{g}$, we now explain how to find an approximate solution of problem (1) at the point $t_{k+1}$ with the given accuracy.

We first compute the local error at the point $t_{k+1}$, by using any classical approach, with the accuracy $\epsilon_{l}$ (see, for example, 3]). After that we determine 
the coefficient of the principal term of the global error by formulas $(6)$. Then we find the maximum step size $\tau_{k}^{*}$ guaranteeing the necessary accuracy 1 :

$$
p=\left[\tau_{k}\left(\left\|\left(\tilde{\psi}_{s}\left(t_{k+1}\right)^{T}, \tilde{\phi}_{s}\left(t_{k+1}\right)^{T}\right)^{T}\right\| / \epsilon_{g}\right)^{1 / s}\right]+1, \quad \tau_{k}^{*}=\tau_{k} / p .
$$

Further, we solve problem (1) on the local interval $\left[t_{k}, t_{k+1}\right]$ by method (2) with the fixed step size $\tau_{k}^{*}$ and obtain the numerical solution at the point $t_{k+1}$ with the accuracy $\epsilon_{g}$. Moreover, in order to compensate the influence of the rejected terms in global error expansion (4) we improve the numerical solution by means of Richardson extrapolation or two RK methods of different orders. Now we set the next step size $\tau_{k+1}=\tau_{k}^{*}$ and repeat all the computations at the next time point, and so on.

In [5], it was shown that, with a practical point of view, it is important to use the stable version of the local-global step size control with an additional bound $\epsilon_{g l}$. To demonstrate the nice practical properties of the stable controller, we apply the Trapezoidal Rule with Newton iteration (TRN method) to the restricted three body problem in differential-algebraic formulation [5].

Table 1. Global errors of the variable step size TRN method with the stable localglobal step size control and with extrapolation $\left(\epsilon_{l}=\epsilon_{g}\right.$ and $\left.\epsilon_{g l}=\epsilon_{g} / 100\right)$

\begin{tabular}{c|ccccc}
\hline Number of & \multicolumn{5}{|c}{ required accuracy } \\
iterations $N$ & $\epsilon_{g}=10^{-03}$ & $\epsilon_{g}=10^{-05}$ & $\epsilon_{g}=10^{-07}$ & $\epsilon_{g}=10^{-09}$ & $\epsilon_{g}=10^{-11}$ \\
\hline 1 & $6.40 \cdot 10^{-04}$ & $8.12 \cdot 10^{-06}$ & $4.74 \cdot 10^{-08}$ & $2.04 \cdot 10^{-10}$ & $2.90 \cdot 10^{-12}$ \\
2 & $6.52 \cdot 10^{-04}$ & $8.06 \cdot 10^{-06}$ & $4.73 \cdot 10^{-08}$ & $2.16 \cdot 10^{-10}$ & $3.62 \cdot 10^{-13}$ \\
\hline
\end{tabular}

From the data of Table 1 it follows that the variable step size TRN method with the stable local-global error control mechanism achieves automatically any reasonable accuracy preassigned for the numerical solution, even when only one Newton iteration per each time point has been fulfilled.

Table 2. Global errors of the variable step size TRN method with the stable localglobal step size control and with extrapolation $\left(\epsilon_{l}=\epsilon_{g}\right.$ and $\left.\epsilon_{g l}=\epsilon_{g} / 100\right)$

\begin{tabular}{c|ccccc}
\hline \multirow{2}{*}{$\begin{array}{c}\text { Number of } \\
\text { iterations } N\end{array}$} & $\epsilon_{g}=10^{-03}$ & $\epsilon_{g}=10^{-04}$ & $\epsilon_{g}=10^{-05}$ & $\epsilon_{g}=10^{-06}$ & $\epsilon_{g}=10^{-07}$ \\
\hline 1 & $1.57 \cdot 10^{+02}$ & $4.43 \cdot 10^{+00}$ & $1.92 \cdot 10^{+00}$ & $2.07 \cdot 10^{+00}$ & $2.00 \cdot 10^{+00}$ \\
2 & $1.85 \cdot 10^{+00}$ & $1.00 \cdot 10^{+00}$ & $1.10 \cdot 10^{-01}$ & $3.47 \cdot 10^{-02}$ & $3.22 \cdot 10^{-03}$ \\
3 & $1.85 \cdot 10^{-01}$ & $1.96 \cdot 10^{-02}$ & $2.19 \cdot 10^{-03}$ & $1.82 \cdot 10^{-04}$ & $3.46 \cdot 10^{-05}$ \\
\hline
\end{tabular}

\footnotetext{
${ }^{1}$ The square brackets in formula (7) means, as is customary, the integral part of the number.
} 
Table 3. Global errors of the variable step size TRN method with the advanced stable local-global step size control and with extrapolation $\left(\epsilon_{l}=\epsilon_{g}\right.$ and $\left.\epsilon_{g l}=\epsilon_{g} / 100\right)$

\begin{tabular}{c|ccccc}
\hline \multirow{2}{*}{$\begin{array}{c}\text { Number of } \\
\text { iterations } N\end{array}$} & $\epsilon_{g}=10^{-03}$ & $\epsilon_{g}=10^{-04}$ & $\epsilon_{g}=10^{-05}$ & $\epsilon_{g}=10^{-06}$ & $\epsilon_{g}=10^{-07}$ \\
\hline 1 & $1.82 \cdot 10^{+00}$ & $1.58 \cdot 10^{+00}$ & $8.87 \cdot 10^{-01}$ & $1.86 \cdot 10^{-01}$ & $3.91 \cdot 10^{-02}$ \\
2 & $1.02 \cdot 10^{-02}$ & $5.92 \cdot 10^{-04}$ & $3.12 \cdot 10^{-05}$ & $1.53 \cdot 10^{-06}$ & $7.41 \cdot 10^{-08}$ \\
3 & $1.02 \cdot 10^{-05}$ & $7.67 \cdot 10^{-08}$ & $1.86 \cdot 10^{-10}$ & $2.47 \cdot 10^{-09}$ & $1.18 \cdot 10^{-09}$ \\
\hline
\end{tabular}

Let us now show that the stable local-global step size control sometimes cannot provide the required accuracy. To illustrate this, we take the TRN method with trivial predictor and apply it to the restricted three body problem on the double interval $[0,2 T]$; i. e. we have increased the length of the integration segment by a factor of 2 . This makes the test problem more difficult for solving with the guaranteed accuracy because of round-off errors and the omitted terms in global error expansion (4). Indeed, the numerical results demonstrate that our error control mechanism cannot provide the required accuracy for any number of Newton iterations per time point (see Table 2).

To improve the local-global step size control in order to solve our test problem with the necessary accuracy, we involve more terms of the global error expansion in the error control procedure. First of all Richardson extrapolation gives us a way to find any reasonable number of the first terms in the local error expansion. Then, by substitution of the coefficients of these terms in the inhomogeneous part of equation (5a), we obtain systems of linear DAEs for the coefficients of relevant terms in the global error expansion. After that we can use the main ideas discussed above in order to compute and control some first leading terms of the global error of Runge-Kutta methods. If we now derive the advanced stable local-global step size control based on the computation of the two first leading terms of the global error expansion and apply it to our test problem then we see that the new advanced version of the step size control is more reliable for practical use (see Table 3). Finally, it is necessary to point out that, as the last example shows, the sufficient quantity of Newton iterations is very important for our step size control to be valid. We refer to [ $[\underline{6}$ for more details.

\section{References}

1. Hairer, E., Wanner, G.: Solving ordinary differential equations II: Stiff and differential-algebraic problems. Springer-Verlag, Berlin, 1996

2. Kulikov, G.Yu.: Convergence theorems for iterative Runge-Kutta methods with a constant integration step. (in Russian) Zh. Vychisl. Mat. Mat. Fiz. 36 (1996) No. 8, 73-89; translation in Comput. Math. Math. Phys. 36 (1996) No. 8, 1041-1054

3. Kulikov, G.Yu.: On the numerical solution of the Cauchy problem for a system of differential-algebraic equations by means of implicit Runge-Kutta methods with a variable integration step. (in Russian) Vestnik Moskov. Univ. Ser. 1 Mat. Mekh. (1997) No. 5, 7-11; translation in Moscow Univ. Math. Bull. 52 (1997) No. 5, 6-10 
4. Kulikov, G.Yu.: A theory of symmetric one-step methods for differential-algebraic equations. Russian J. Numer. Anal. Math. Modelling. 12 (1997) No. 6, 501-523

5. Kulikov, G.Yu.: A local-global version of a stepsize control for Runge-Kutta methods. Korean J. Comput. Appl. Math. 7 (2000) No. 2, 289-318

6. Kulikov, G.Yu.: One-step methods and implicit extrapolation technique for index 1 differential-algebraic systems. Russian J. Numer. Anal. Math. Modelling. (to appear) 